\title{
Potencial de producción de energía eléctrica en México empleando la circulación del parque vehicular
}

\section{Potential of electricity production in Mexico using the circulation of the vehicle fleet}

\author{
CASTAÑEDA-OLIVARES, Felipe†** \& AGUIRRE-RODRÍGUEZ, Claudia
}

Universidad Tecnológica de San Juan del Río. Av. La Palma 125. Col. Vista Hermosa. San Juan del Río, Qro. C.P. 76800

ID $1^{\mathrm{er}}$ Autor: Felipe, Castañeda-Olivares / ORC ID: 0000-0003-0510-1591, Researcher ID Thomson: X-3289-2019, CVU CONACYT ID: 231030

ID $1^{\text {er }}$ Coautor: Claudia, Aguirre-Rodríguez / ORC ID: 0000-0003-4476-606X, Researcher ID Thomson: X-3310-2019

DOI: $10.35429 / J T E N .2019 .9 .3 .25 .30$

Recibido 03 de Enero, 2019; Marzo 30 Marzo, 2019

\begin{abstract}
Resumen
La producción de energía eléctrica es una necesidad de la vida moderna y México no se escapa de la misma. México se encuentra en el lugar 51 del Índice de Competitividad Global en materia de electricidad, según estudios del Foro Económico Mundial. En donde se usan las siguientes fuentes de producción energética. De Tecnología Convencional: Ciclo combinado, Termoeléctrica Convencional, Carboeléctrica, Turbo gas, Combustión Interna, Nucleoeléctrica. Energía Limpia y Renovable: Hidroeléctrica, Eólica, Geotérmica, Solar Fotovoltaica y Termo Solar. También se importa energía eléctrica de los Estados Unidos. La presente investigación tiene como objetivo dar a conocer otras posibilidades de generación de energía eléctrica que no han sido exploradas en México ni contempladas en el Programa de Desarrollo del Sistema Eléctrico Nacional (PRODESEN, 2019-2033). La hipótesis planteada como propuesta es que se pueden usar los 38 millones de vehículos automotores que existen y circulan por los caminos y carreteras del país para generar electricidad por medio de generadores piezoeléctricos y aerogeneradores. Con base en las estimaciones del escenario de planeación, la demanda máxima integrada del Sistema Interconectado Nacional (SIN) proyecta un crecimiento medio anual de $3.2 \%$ entre 2019 y 2033. Para lograr este crecimiento es necesario considerar todas las posibilidades de producción de energía y su rentabilidad.
\end{abstract}

Efecto piezoeléctrico, Aerogeneradores, Energía limpia

\begin{abstract}
The production of electricity is a necessity of modern life and Mexico does not escape it. Mexico ranks 51st in the Global Electricity Competitiveness Index, according to World Economic Forum studies. Where the following sources of energy production are used. Conventional Technology: Combined Cycle, Conventional Thermoelectric, Carb, Turbo Gas, Internal Combustion, Nuclear Power. Clean and Renewable Energy: Hydroelectric, Wind, Geothermal, Solar Photovoltaic and Solar Thermal. Electrical power is also imported from the United States. The objective of this research is to make known other possibilities of generating electricity that have not been explored in Mexico or contemplated in the Program for the Development of the National Electrical System (PRODESEN, 2018-2032). The hypothesis put forward as a proposal is that the 38 million motor vehicles that exist and circulate on the country's roads and highways can be used to generate electricity through piezoelectric generators and wind turbines. Based on the planning scenario estimates, the maximum integrated demand of the National Interconnected System (SIN) projects an average annual growth of $3.2 \%$ between 2018 and 2032. To achieve this growth, it is necessary to consider all the possibilities of energy production and its profitability.
\end{abstract}

Piezoelectric effect, Wind turbines, Clean energy

Citación: CASTAÑEDA-OLIVARES, Felipe \& AGUIRRE-RODRÍGUEZ, Claudia. Potencial de producción de energía eléctrica en México empleando la circulación del parque vehicular. Revista de Ingeniería Tecnológica. 2019. 3-9: 25-30

\footnotetext{
* Correspondencia del Autor (Correo electrónico: fcastanedao@utsjr.edu.mx)

$\dagger$ Investigador contribuyendo como primer autor.
} 


\section{Introducción}

La energía eléctrica en el mundo es un insumo primordial y estratégico para el desarrollo de la sociedad. La forma de producirla es un tema que ha cambiado en México en los últimos años. Como resultado de la Reforma energética en México, ahora en México se produce electricidad en un régimen de libre competencia. Sin embargo, la Secretaría de Energía (SENER) es la responsable de formular el programa sectorial para el desarrollo de la industria eléctrica. Dicho programa se ve plasmado en el Programa de Desarrollo del Sistema Eléctrico Nacional (PRODESEN), con un horizonte de 15 años 2019-2033.

Existen en el mundo muchas tecnologías para la producción de energía eléctrica, sin embargo, no todas se han implementado en México. Para poder cubrir la demanda del futuro de los mexicanos se requiere crecer a un ritmo de $3.2 \%$ anual en materia de generación de energía eléctrica. (Secretaría de Energía, 2019)

A través de la Ley de Transición Energética en materia de energías renovables, México estableció metas y obligaciones para las áreas de energías limpias y eficiencia energética. También los actores que deberán generar los reglamentos, programas y demás lineamientos que detallen las acciones e instrumentos para asegurar la correcta operación del sistema en estas áreas, principalmente, la Secretaría de Energía (Sener), la Comisión Reguladora de Energía (CRE), el Centro Nacional de Control de Energía (Cenace) y la Comisión Nacional para el Uso Eficiente de la Energía (Conuee). En los transitorios de la Ley se mantienen las metas establecidas en leyes anteriores sobre el porcentaje de energías limpias en la generación eléctrica: $25 \%$ en $2018,30 \%$ en 2021 , y $35 \%$ en 2024. (Mulás del Pozo, 2019)

\section{Justificación}

Aun cuando el PRODESEN contempla en su planeación las alternativas de producción de energía eléctrica para un horizonte de 15 años, consideramos pertinente analizar una alternativa sustentable, no contaminante y que podría cambiar el rumbo de la estrategia en la producción de electricidad en algunas regiones del país.

\section{Problema de investigación}

La posible implementación en la generación de energía eólica y piezoeléctrica ubicadas en las autopistas de México y sus ciudades.

\section{Objetivos}

\section{Objetivo General}

Determinar la viabilidad en México del uso de generadores de electricidad piezoeléctricos y aerogeneradores a usarse en carreteras y ciudades de México.

\section{Objetivos específicos}

- Investigar el estado de la producción de electricidad en México.

- Investigar el plan de crecimiento de la producción eléctrica a futuro.

- Investigar el funcionamiento de los generadores piezoeléctricos y aerogeneradores y proponer los lugares aptos para su uso en México.

\section{Marco Teórico}

\section{La piezoelectricidad}

La piezoelectricidad es un fenómeno eléctrico que se presenta en algunos minerales y fue descubierto por los hermanos Curie (J. Curie, 1880; P. Curie, 1880) a finales del siglo xix. Sin embargo, solo hasta el año de 1916 se dio a conocer su primera aplicación en el trabajo de Paul Langevin y Constantin Chilowski en la construcción de un detector ultrasónico utilizado para la medición de los fondos marinos y la detección de submarinos durante la Primera Guerra Mundial.

Según la bibliografía relacionada con la generación de energía a partir de celdas piezoeléctricas, en 1944 los científicos B.M. Wool e I.P. Goldman, del Lebedev Physycal Institute, fueron los primeros en implementar un método de sinterización de cerámicas piezoeléctricas con titanato de bario (BaTiO3). (Leyton Vásquez \& Roa Becerra, 2017) 
En los últimos años se ha despertado el interés por el aprovechamiento de la energía cinética y potencial presente en el tráfico vehicular, en parte por la necesidad de contar con dispositivos energéticamente autónomos, tales como lámparas para alumbrado público, semáforos, sensores de tráfico, cámaras de vigilancia, entre otros. (Castro S. \& Arias C., 2015)

Se han realizado experimentos con generadores piezoeléctricos tales como la realizada en el año 2009 por una firma de ingeniería de Israel, quienes fueron noticia por el desarrollo de una nueva técnica que permite generar electricidad a partir del peso, movimiento, y vibraciones de los vehículos; Actualmente ya hay generadores específicos para carreteras, vías férreas, pistas de aterrizaje y circulación de peatones. El producto con mayor cantidad de pruebas ha sido el de los generadores piezoeléctricos que se instalan debajo del asfalto, los generadores se instalan $0.05 \mathrm{~m}$ por debajo de la rasante, en este caso la presión de los vehículos sobre los dispositivos se convierte en electricidad, abasteciendo de esta manera los alumbrados públicos en las zonas de influencia de las carreteras. (Tamayo Zapata \& Cardozo Gutierrez, 2017)

En el año 2007 surgió la empresa Innowattech, pionera en el desarrollo de esta tecnología en las vías. Esta es una de las más conocidas a nivel mundial por sus múltiples productos piezoeléctricos, esta empresa desarrollo un método de obtención de electricidad que utiliza la energía mecánica producida por la vibración de vehículos. Se centra en los actuadores piezoeléctricos de alto rendimiento, los cuales son unos dispositivos capaces de utilizar los cambios de presión producidos por las vibraciones y el movimiento para realizar la trasformación de la energía y obtener electricidad. Este efecto es conocido como piezo-mecánico comprimido. (Figura 1)

Estos piezoeléctricos se instalan bajo la capa de pavimento asfaltico, la energía generada es utilizada para la iluminación de las vías y para monitorizar los datos de circulación. (Tamayo Zapata \& Cardozo Gutierrez, 2017)

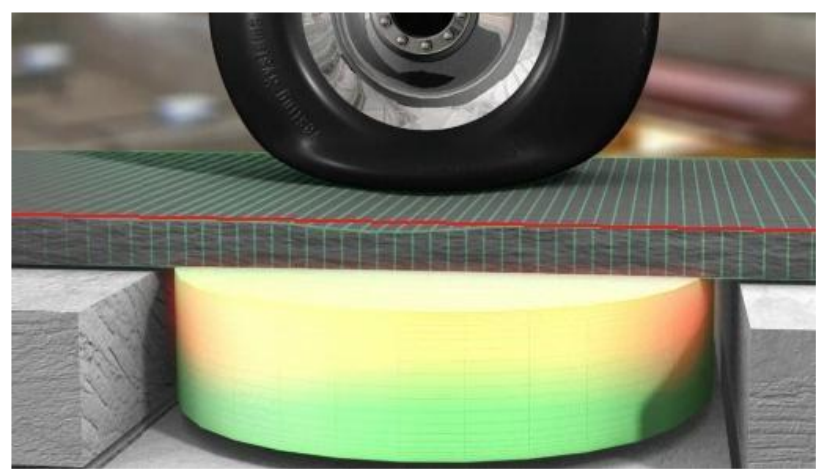

Figura 1 Deformación vertical asociado con el paso de la rueda

\section{Aerogeneradores}

$\mathrm{Si}$ los generadores piezoeléctricos son prometedores, la presión del aire en forma de vientos con mayor velocidad acumulan mucha más energía. Todos los vehículos en las vías rápidas generan olas de presión de diferente forma $\mathrm{y}$ velocidad que bien pueden ser aprovechados en aerogeneradores. En el mundo existen varios diseños de ellos, pero entre los más representativos están los Darrieus-Savonius desarrollados en Taiwan (Figura 2)
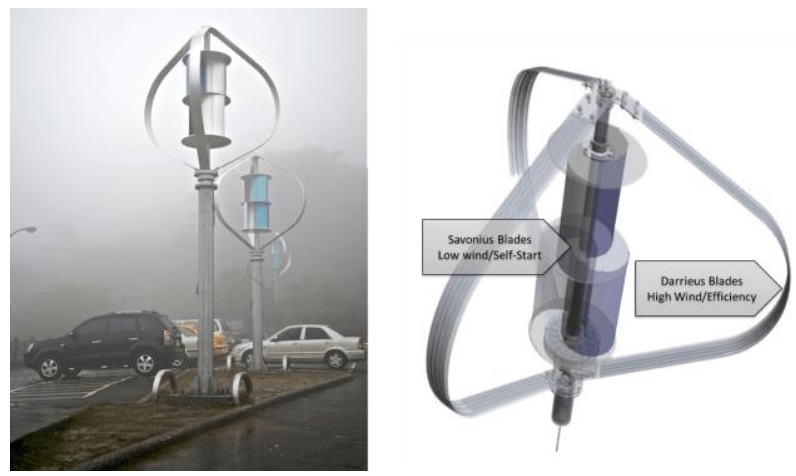

Figura 2 Turbina Darrieus-Savonius

Este proyecto se amortizó en menos de cuatro años con una vida estimada de diez. Es decir, el $60 \%$ de su vida útil es un generador de energía limpia y a costo cero. Estos molinos no solo captan el viento generado por los coches al circular a altas velocidades, sino cualquier ráfaga. Se pueden instalar a lo largo de la mediana de una autopista (donde el acceso es muy sencillo y económico). (Marcos, 2016)

Dentro de las ventajas que tienen es que a nivel medioambiental su impacto es muy bajo:

Usan un espacio ya inutilizado, por lo que no requieren invadir grandes extensiones de campo; 
- $\quad$ Su ruido es prácticamente nulo, sobre todo comparado con el del tráfico;

- El peligro de herir a la fauna, especialmente a las aves, es mucho menor debido a la baja velocidad del giro de las aspas;

Las sinergias que crea con líneas de tensión de media tensión de localidades cercanas podrían incluso retirar el tendido eléctrico actual. (Marcos, 2016)

\section{Resultados}

\section{Métodos de producción de energía eléctrica}

Existen los siguientes sistemas de producción de energía eléctrica y sus \% de capacidad (Tabla 1) efectiva instalada (31 dic 2018) en México. (Secretaría de Energía, 2019)

\begin{tabular}{|l|l|}
\hline \multicolumn{2}{|c|}{ Convencional } \\
\hline Ciclo combinado & $36.5 \%$ \\
\hline Termoeléctrica convencional & $17 \%$ \\
\hline Carboeléctrica & $7.7 \%$ \\
\hline Turbogás & $4.6 \%$ \\
\hline Combustión Interna & $1 \%$ \\
\hline \multicolumn{2}{|c|}{ Limpia } \\
\hline Bioenergía & $0.5 \%$ \\
\hline Hidroeléctrica & $18 \%$ \\
\hline Eólica & $6.8 \%$ \\
\hline Geotérmica & $1 \%$ \\
\hline Solar Fotovoltaica & $2.6 \%$ \\
\hline Nucleoeléctrica & $2.3 \%$ \\
\hline
\end{tabular}

Tabla 1 Capacidad Instalada efectiva por tipo de tecnología (70,053 MW)

Como se puede observar en la Tabla 1, la producción de energía eléctrica convencional sigue siendo la principal fuente de producción de energía eléctrica, aunque en años recientes se busca incrementar la producción "limpia", con el fin de cumplir con las disposiciones de la Ley de Transición Energética en materia de energías renovables.

\section{Datos relevantes de la situación actual en México}

Al año 2015, según el censo realizado por el Instituto Nacional de Estadística y Geografía (INEGI) se estima una población de aproximadamente $119,938,473$ personas que residen en el país. Y según la SENER y la SIE al año de 2017, se estimó una población de 123.518 millones de habitantes. De las cuales, una parte cuenta con un vehículo particular, y circulan diariamente por carreteras y autopistas del país.
A nivel país existen más de 38 millones de vehículos automotores registrados. (INEGI, 2015) Con la cantidad de vehículos existentes en México, las mejores opciones para implementar generadores eléctricos piezoeléctrico y aerogeneradores son:

\begin{tabular}{|l|r|}
\hline Autopista & Longitud km \\
\hline México-Puebla & 130 \\
\hline México- Querétaro & 260 \\
\hline México-Acapulco & 367 \\
\hline México-Nogales & 2378 \\
\hline México-Cuernavaca & 85 \\
\hline Carretera Panamericana & 1920 \\
\hline \multicolumn{2}{|c|}{ Ciudades más pobladas } \\
\hline México & Puebla \\
\hline Guadalajara & Tijuana \\
\hline Monterrey & Zapopan \\
\hline
\end{tabular}

Tabla 2 Autopistas más usadas y Ciudades Más pobladas de México (Monroy, 2018)

Cada una de las autopistas mencionadas supera los 600 vehículos por hora. Además, las ciudades con mayor población humana $\mathrm{y}$ vehicular tienen un alto potencial en las calles y cruceros que por lo general están saturados. En el período 2019-2033, se estima que el PIB de los sectores Agrícola y Servicios crecerá 2.7\%, mientras que el industrial $2.9 \%$. Se prevé que, en 2033, el sector Agrícola representará el 3.3\% del PIB Nacional, mientras que, el Industrial y los Servicios integrarán el $31.3 \%$ y $65.4 \%$ respectivamente. Además, se estima que el consumo energético crezca $3.5 \%$, a un ritmo mayor que el PIB que se estima en el $2.8 \%$. (Secretaría de Energía, 2019). Por tanto, se observa un déficit que de alguna manera habrá de cubrirse.

\section{Estrategias a nivel mundial}

Innowattech menciona que "Una sola pista equipada con el generador piezoeléctrico ya está proporcionando a los israelíes 0,5 megavatios por hora, suficiente para abastecer a 600 hogares durante un mes" Estos datos son alentadores en la búsqueda de alternativas en la producción de electricidad. El costo promedio que estima la empresa israelí para la implementación de los generadores oscila los US\$ $650 \mathrm{mil}$ por $\mathrm{km}$, teniendo una equivalencia en energía correspondiente a US\$ 6,5 mil por kilovatio $(\mathrm{kW})$, no obstante la administración de Innowattech afirma: "el costo debe caer un $60 \%$ cuando llegan a la producción en masa, haciendo el sistema más barato que la energía solar" (Tamayo Zapata \& Cardozo Gutierrez, 2017) 
Tanto en Europa como en Norteamérica están trabajando en otras alternativas para disminuir los gases de efecto invernadero y no es precisamente el tema en cuestión, pues se han dado cuenta que es un conjunto de técnicas y acciones las que pueden resolver el problema ambiental. Tal es el caso del uso de aluminio en la construcción de vehículos más ligeros (Palazzo \& Geyer, 2019) (Milovanoff, y otros, 2019)

También los japoneses están promoviendo integrar los vehículos eléctricos a la red de energía eléctrica. Con los generadores propuestos aquí podría hacerse más fácil (Iwafune, Ogimoto, \& Azuma, 2019). La Unión Europea también está intentando reducir la emisión de los gases de efecto invernadero para combatir el cambio climático y una de sus alternativas es el uso del biometano. (Goulding, Fitzpatrick, O'Connor, Browne, \& Power, 2019)

\section{Perspectivas de implementación de nuevas fuentes de energía}

Según un estudio realizado por Toyota, con un solo kilómetro en un solo carril con esta tecnología piezoeléctrica, se podrían producir electricidad suficiente para dar energía a unos 300 hogares. (Toyota, 2017)

En cuanto a los aerogeneradores, este tipo de parques eólicos distribuidos podrían ser el futuro, debido a sus ventajas frente a los grandes molinos (de 5 a $10 \mathrm{MW}$ ). Especialmente en lo económico por transporte o mantenimiento. Un gran molino averiado implica un gran desembolso, y su reparación requiere tiempo y recambios de elevado coste. Mientras que un molino pequeño puede ser cambiado por otro en cuestión de horas. (Marcos, 2016)

\section{Conclusiones}

En el caso de los generadores piezoeléctricos, coincido con Tamayo y Cardozo (2017) quienes afirman que la energía que se produce por el aprovechamiento de la energía captada por el tránsito de los vehículos a lo largo de los paneles instalados estaría disponible a lo largo del día gracias a la afluencia de vehículos en los perfiles viales donde se podría instalar esta tecnología, a diferencia de otras energías renovables que están sometidas a ciclos de producción.
La tecnología de Innowattech plantea que en una vía de $1 \mathrm{~km}$ carril de carretera se pueden producir $250 \mathrm{KWh}$, este valor fue obtenido con base en generadores que están incrustados a lo largo de la vía en dos filas. Con un tránsito aprox. a 600 vehículos pesados por hora. El costo para $1 \mathrm{~km}$ de instalación está en US\$650,250 que cubre la implementación del sistema en cuanto a los sistemas de recolección, almacenamiento, distribución y obra civil.

En México el flujo vehicular ronda los 2,200 vehículos por hora. Con un gran porcentaje de vehículos pesados ya que la carga por ferrocarril no se ha desarrollado a la par de la economía y se ha priorizado el transporte de materiales por carretera. Lo cual significa un alto potencial de generación de energía eléctrica por este concepto. Aunado a que los sistemas piezoeléctricos son un sistema eficiente $\mathrm{y}$ amigable con el ambiente y el entorno donde sea instalado, siendo fuentes de energías alternativas y sostenibles, con una múltiple opción en su forma e instalación.

Las referencias consultadas coinciden en los requisitos para la implementación de los generadores piezoeléctricos o de aerogeneradores.

- Alto tráfico de vehículos pesados complementado con un volumen mixto vehicular.

Un paso constante por el punto donde se han instalado los piezoeléctricos.

Ancho de la vía donde se instalará el sistema.

Corrientes de aire naturales.

Una de las grandes ventajas de ambos sistemas es la supresión del coste del transporte de electricidad. Ya que se usaría en las áreas cercanas o en las mismas vías de comunicación. El tiempo dedicado a investigación y a comercializar las energías renovables ha sido poco, comparado con el uso de otras energías convencionales y la tendencia es que se sigan abaratando los costos a través del tiempo, por lo que las carreteras tendrán mucho que decir en el futuro de la energía en el mundo y la propuesta es que México se integre a todas las formas de producción de energía eléctrica. Sobre todo porque tiene los medios para hacerlo desde la perspectiva de la inversión privada o gubernamental. 


\section{Referencias}

Castro S., D., \& Arias C., E. (2015). Análisis y diseño de un prototipo suelo generador de energía eléctrica utilizando materiales piezoeléctricos. En E. Serna, Avances en Ingeniería (págs. 315-320). Medellín, Antioquía, Colombia: Instituto Antioqueño de Investigación. Obtenido de https://www.researchgate.net/profile/Edgar_Ser na_M/publication/331385300_Avances_en_Ing enieria/links/5c76e2d8299bf1268d2b0152/Ava nces-en-Ingenieria.pdf\#page $=316$

Goulding, D., Fitzpatrick, D., O'Connor, R., Browne, J., \& Power, N. (2019). Introducing gaseous transport fuel to Ireland: A strategic infraestructure framework. Renewable Energy(136), 549-557.

INEGI. (05 de 11 de 2015). Instituto Nacional de Estadísitica y Geografía. Recuperado el 10 de 09 de 2019, de https://www.inegi.org.mx/temas/ estructura/

Iwafune, Y., Ogimoto, K., \& Azuma, H. (2019). Integration of Electric Vehicles into the Electric Power System Based on Results of Road Traffic Census. Energies, 12(10), 1849.

Leyton Vásquez, H., \& Roa Becerra, N. (2017). Representación matemática y Técnicas de caracterización para celdas piezoeléctricas, una revisión bibliográfica. unminuto.edu, EneroJunio(22), 66-75.

Marcos, M. (2 de Noviembre de 2016). CONSTRUCCIÓN. Obtenido de https://blog.ferrovial.com/es/2016/11/generarelectricidad-con-coches/

Milovanoff, A., Chul Kim, H., De Kleine, R., Wallington, T. J., Posean, I., \& MacLean, H. L. (2019). A dynamic fleet model of U.S light-duty vehicle lightweighting and associated greenhouse gas emissions from 2016-2050. Environmental science \& technology,, 53(4), 2199-2208.

Monroy, S. (21 de Septiembre de 2018). Las carreteras más importantes de México. El Universal. Obtenido de https://www.eluniversal.com.mx/ autopistas/lascarreteras-mas- importantes-de-mexico
Mulás del Pozo, P. (01 de Agosto de 2019). revista comercio exterior.com. Recuperado el 2019,

de

https://www.revistacomercioexterior.com/articu lo.php?id=133\&t=el-sector-energetico-y-la-leyde-transicion-energetica

NOTIMEX. (29 de Enero de 2018). MILENIO. Obtenido de https://www.milenio.com/negoci os/numero-autos-particulares- crece-mexicosuman-29-4- millones

Palazzo, J., \& Geyer, R. (2019). Consequential Life Cycle Assessment of Automotive Material Substitution: Replacing Steel with Aluminum in Production of North American Vehicles. Environmental Impact Assessment Review, 1-39.

Secretaría de Energía. (2019). Programa de Ampliación y Modernización de la Red Nacional de Transmisión y Redes Generales de Distribución del Mercado Eléctrico Mayorista. CENACE, Centro Nacional de Control de Energía (CENACE). México: Secretaría de Energía. Recuperado el 01 de septiembre de 2019 , https://www.gob.mx/sener/documentos/prodese n-2019-2033

Tamayo Zapata, D. A., \& Cardozo Gutierrez, N. K. (2017). EL USO DE PIEZOELÉCTRICOS PARA LA GENERACIÓN DE ENERGÍA SOSTENIBLE COMO PROYECTO PILOTO EN UN PERFIL VIAL DE BOGOTÁ. Bogotá: Universidad Católica de Colombia.

Toyota. (1 de Marzo de 2017). Toyota. Obtenido de Carreteras que generan electricidad: https://www.toyota.es/world-of-toyota/articlesnews-

events/2017/carreteras_generaran_electricidad 